\title{
Plasma olfactomedin 4 level in peripheral blood and its association with clinical features of breast cancer
}

\author{
CHAOQIAN LIU ${ }^{1 *}$, YAN GUO $^{2^{*}}$, WEIWEI WU ${ }^{3 *}$, ZHENZHEN ZHANG $^{1,3}$, LU XU $^{1}$, KAINAN WU $^{1}$, \\ WEI HU ${ }^{1}$, GUOPING LIU ${ }^{1}$, JUNYI SHI ${ }^{1}$, CHENG XU ${ }^{3}$, JIANWEI BI ${ }^{4}$ and YUAN SHENG ${ }^{1}$ \\ Departments of ${ }^{1}$ Breast Surgery and ${ }^{2}$ Endocrinology, Changhai Hospital, Second Military Medical University, \\ Shanghai 200433; ${ }^{3}$ Biotecan Medical Diagnostics Co., Ltd, Zhangjiang Center for Translational Medicine, Shanghai 200120; \\ ${ }^{4}$ Department of General Surgery, Changhai Hospital, Second Military Medical University, Shanghai 200433, P.R. China
}

Received March 24, 2016; Accepted August 15, 2017

DOI: $10.3892 / \mathrm{ol} .2017 .7193$

\begin{abstract}
The present study aimed to investigate the expression of olfactomedin 4 (OLFM4) in plasma of patients with breast cancer and its association with diagnosis, metastasis and prognosis of breast cancer. OLFM4 gene expression level of peripheral blood plasma in 60 patients with breast cancer and 26 healthy donors was examined by ELISA. The expression of OLFM4 in tumor tissues of patients with breast cancer was evaluated by immunohistochemistry (protein expression) and reverse transcription-quantitative polymerase chain reaction (mRNA expression), respectively. Circulating tumor cells (CTCs) were detected in a certain set of patients. The expression of OLFM4 in plasma of the overall healthy people was higher compared with patients with breast cancer. The plasma OLFM4 level in patients with breast cancer was consistent with the expression of OLFM4 protein in tumor tissues $\left(\mathrm{R}^{2}=1\right)$, indicating that the level of plasma OLFM4 expression may represent the expression of OLFM4 in breast cancer tissues. The plasma OLFM4 level in patients with histological grade I was significantly lower compared with grade III $(\mathrm{P}<0.05)$. Breast cancer patients with positive CTC were associated with low level of plasma OLFM4. These results suggest that low OLFM4 expression in plasma or tissue specimens of breast cancer patients is more likely to represent low histological differentiation and decreased invasive/metastatic capabilities. Taken together, plasma OLFM4 level may be considered as a
\end{abstract}

Correspondence to: Dr Yuan Sheng, Department of Breast Surgery, Changhai Hospital, Second Military Medical University, 168 Changhai Road, Shanghai 200433, P.R. China

E-mail: shengyuan_smmu@163.com

Dr Jianwei Bi, Department of General Surgery, Changhai Hospital, Second Military Medical University, Shanghai 200433, P.R. China E-mail: bijianwei@medmail.com.cn

\section{${ }^{*}$ Contributed equally}

Key words: breast cancer, olfactomedin 4, circulating tumor cells, plasma biomarker for diagnosis and prognosis of breast cancer for cases where there are difficulties in obtaining tumor tissue samples.

\section{Introduction}

The olfactomedin 4 (OLFM4; GW112; hGC-1) gene was first cloned from human myeloblasts and is located on human chromosome 13 (13q14.3). The encoded protein product, OLFM4, is a member of the olfactomedin-domain family with intracellular localization mostly in the mitochondria. OLFM4 is characterized by a coiled-coil domain at the $\mathrm{N}$-terminus and a conserved olfactomedin domain of $\sim 250$ amino acids at the C-terminus (1). OLFM4 commonly exists in gastrointestinal epithelial cells, prostate and bone marrow $(2,3)$, where it participates in cellular processes, including proliferation, apoptosis, metabolism and differentiation (4).

High OLFM4 expression has been detected at the early stages of adenoid and colorectal tumors. OLFM4 expression is regulated by the Ras-nuclear factor- $\kappa \mathrm{B} 2$ signaling pathway, one of the main tumorigenic pathways, and has important associations with tumor development and progression (4). Research has demonstrated that OLFM4 is involved in regulation of cell apoptosis, where OLFM4 attenuates apoptosis induced by hydrogen peroxide and tumor necrosis factor- $\alpha$, and also inhibits the release of cytochrome $\mathrm{c}$ and activation of caspase 3 and 9. OLFM4 protein in cells ultimately has an anti-apoptotic role when cells are treated with a combination of interferon (IFN) $\beta$ and retinoic acid $(5,6)$. Furthermore, OLFM4 exerts a regulatory role in the cell cycle in DNA synthesis and mitosis from the $\mathrm{S}$ to the $\mathrm{G} 2 / \mathrm{M}$ phase (7). In 2011, Besson et al (5) analyzed colorectal tumor tissue at different stages by quantitative immunohistochemistry. It was found that OLFM4 is over-expressed in low-grade and high-grade adenoma tumor tissues, adenoma in situ, and stage I-II colorectal cancer tissues. By contrast, there were no significant differences in OLFM4 expression between stage III-IV colorectal cancer tissue and normal colon crypt tissue. Liu et al (8) reported reduced expression of OLFM4 in patients with advanced colon cancer (stages III and IV), lymph node metastases and distant metastases compared with normal colon tissue. Using a mouse melanoma model, Park et al (9) observed that OLFM4 suppresses tumor growth and metastasis by downregulating 
integrin and matrix metalloproteinase gene expression. Therefore, OLFM4 may have a dual role in tumor cell growth depending on the stage and molecular characteristics of tumor progression.

Studies have shown relatively low levels of OLFM4 expression in normal tissues of several organs, including the stomach, colon and pancreas. Whereas, to varying degrees, upregulation of OLFM4 expression has been implicated in gastric, pancreatic, lung, colon, rectal, and head/neck squamous cell cancer $(5,10-12)$. A study observed that there was no OLFM4 expression prostate cancer tissue, with moderate expression in benign prostatic hyperplasia and high expression in normal prostate tissue (13). Abnormal OLFM4 expression exists in a variety of tumors, but associated research findings are inconsistent, perhaps owing to the cell and tissue specificity of OLFM4.

The majority of studies on OLFM4 are based on gene or protein expression analysis in tumor tissue specimens. Such research is largely limited, as in certain cases, it can be difficult to collect clinical specimens from surgery. Clinical relevance of OLFM4 expression levels in peripheral blood mononuclear cells and circulating tumor cells in pancreatic cancer has been demonstrated (14). As OLFM4 expression levels are significantly increased in PBMCs of pancreatic cancer patients, OLFM4 expression can be used as an indicator for pancreatic cancer in early diagnosis and for prognostic determination (14). Additionally, colon cancer studies show that OLFM4 upregulation and associated gene expression in CTCs are significantly associated with liver metastasis of tumors $(15,16)$. OLFM4 is a secreted glycoprotein and research, which assesses the clinical significance of serum/plasma OLFM4 levels in the peripheral blood of tumor patients, is limited. A study of serum OLFM4 levels in the peripheral blood of gastric cancer patients (11) suggested that OLFM4 expression in peripheral blood could be used as an indicator in early diagnosis and prognostic determination of gastric cancer. To date, to the best of our knowledge, no reports have been published on serum/plasma OLFM4 expression patterns in other tumors, including breast cancer.

Breast cancer remains the second leading cause of tumor mortality in women. At the gene level, breast cancer is a highly heterogeneous disease. Koshida et al (10) reported that OLFM4 expression levels are significantly higher in $69 \%$ of breast cancer tissues compared with the corresponding adjacent normal tissues. This percentage is lower compared with the values reported in colon $(90 \%)$ and lung $(85 \%)$ cancer tissue specimens over the same period (10). Furthermore, it was thought that the OLFM4 expression level has no significant association with common clinical variables $(9,10)$. However, a limited number of studies have reported OLFM4 gene expression in breast cancer patients, and the expression levels of this gene in the peripheral blood of tumor patients remain to be documented $(1,10,11,17)$. To assess the association of OLFM4 gene expression with the development and progression of breast cancer, breast cancer tissue and peripheral blood specimens were selected from patients of different histological grades, and these specimens were used to detect OLFM4 expression in the present study. The results were analyzed to determine the specificity of the OLFM4 gene for diagnosis of breast cancer or prognostic determination in patients with breast cancer.

\section{Materials and methods}

Enrollment and grouping. The patients were enrolled at the Department of Thyroid and Breast Surgery at the Changhai Hospital, which is affiliated with The Second Military Medical University (Shanghai, China) from February 2014 to February 2015. The present study was approved by the Ethics Committee of the Changhai Hospital of Shanghai. All enrolled patients signed informed consent prior to the study. All patients enrolled were female and aged between 22 and 87 years (mean, 56 years). The tumor-node-metastasis (TNM) stage was classified (18). Peripheral blood specimens were extracted from 54 patients with breast cancer, 6 patients with benign breast tumors, and 26 healthy individuals (4-5 ml each). Samples were taken prior to any treatment when the patients first arrived at the hospital. Patients typically were presented with histologically confirmed invasive ductal carcinoma. The general characteristics of the patients are listed in Table I. The levels of OLFM4 gene expression in the two groups were measured by ELISA. Additionally, $10 \mathrm{mg}$ of tumor tissue and normal adjacent tissue was obtained from 24 patients with breast cancer, and control tissue specimens were obtained from another 10 healthy individuals. The tissue specimens were used for RNA extraction and quantitative analysis of OLFM4 mRNA levels. Moreover, paraffin sections were prepared using tumor tissue specimens from 39 patients with breast cancer, tissues were fixed with $10 \%$ formalin for $24 \mathrm{~h}$ at room temperature. Fixed tissues were placed in embedding cassettes and cut at $5 \mu \mathrm{m}$. OLFM4 expression in tissue specimens of human breast cancer was detected by immunohistochemistry. A total of 42 patients and 5 benign samples were subjected to peripheral blood collection and underwent circulating tumor cell (CTC) detection by immunostaining-fluorescence in situ hybridization (iFISH) and subtraction enrichment $(19,20)$, and 31 patient samples and 5 benign samples underwent CTC detection using the CellSearch system, as previously described (21).

Sandwich ELISA analysis. An olfactomedin 4 (OLFM4) ELISA kit (cat. no. 027220; United States Biological, Salem, MA, USA) was used to measure the plasma concentration of olfactomedin 4. The microtiter plate provided in the ELISA kit has been pre-coated with an antibody specific to OLFM4. A total of 9 diluted standards were prepared, including 4,000, $2,000,1,000,500,250,125,62.5,32.25$ and $0 \mathrm{pg} / \mathrm{ml}$. Detection in all 86 samples was performed following 100-fold dilution and the samples were tested in triplicate. The main detection process included a number of steps. Initially, all reagents, samples and standards were prepared and $100 \mu \mathrm{l}$ of each diluted standard or samples were added to the appropriate microtiter plate wells with a biotin-conjugated antibody preparation specific to OLFM4. Following incubation for $2 \mathrm{~h}$ at $37^{\circ} \mathrm{C}$, the solution in each well was removed without any subsequent washing steps. A total of $100 \mu 1$ Detection Reagent A working solution was added to each well followed by incubation for $1 \mathrm{~h}$ at $37^{\circ} \mathrm{C}$ with the plate sealed. Subsequently, the solution was aspirated, and the wells were washed with wash solution. Then, $100 \mu$ l Detection Reagent B working solution was added to each well and the samples were incubated for $30 \mathrm{~min}$ at $37^{\circ} \mathrm{C}$. The solution was aspirated and the wells were 
Table I. Plasma OLFM4 expression level and characteristics of patients with breast cancer.

\begin{tabular}{|c|c|c|}
\hline \multirow[b]{2}{*}{ Parameters } & \multicolumn{2}{|c|}{ OLFM4 expression } \\
\hline & $\begin{array}{l}\leq \text { mean, } \\
\mathrm{ng} / \mathrm{ml}(\mathrm{n})\end{array}$ & $\begin{array}{l}>\text { mean, } \\
\mathrm{ng} / \mathrm{ml}(\mathrm{n})\end{array}$ \\
\hline \multicolumn{3}{|l|}{ Samples } \\
\hline Breast cancer & $32(54)$ & $22(54)$ \\
\hline Benign tumor & $3(6)$ & $3(6)$ \\
\hline Healthy donor & $15(26)$ & $11(26)$ \\
\hline \multicolumn{3}{|c|}{ Histological grade } \\
\hline I & $9(19)$ & $10(19)$ \\
\hline II & $12(21)$ & $9(21)$ \\
\hline III & $9(14)$ & $5(14)$ \\
\hline \multicolumn{3}{|c|}{ Lymph node status } \\
\hline Positive & $16(25)$ & $9(25)$ \\
\hline Negative & $16(28)$ & $12(28)$ \\
\hline \multicolumn{3}{|c|}{ Progesterone/estrogen receptor } \\
\hline Positive & $24(38)$ & $14(38)$ \\
\hline Negative & $8(15)$ & $7(15)$ \\
\hline \multicolumn{3}{|l|}{ TNM stage } \\
\hline $\mathrm{I}$ & $12(22)$ & $10(22)$ \\
\hline II & $16(22)$ & $3(22)$ \\
\hline III & $3(8)$ & $5(8)$ \\
\hline IV & $0(1)$ & $0(1)$ \\
\hline \multicolumn{3}{|c|}{ OLFM4 expression by IHC } \\
\hline Negative (0) & $1(1)$ & $0(1)$ \\
\hline Positive (1+) & $3(6)$ & $3(6)$ \\
\hline Positive (2+) & $9(13)$ & $4(13)$ \\
\hline Positive (3+) & $5(8)$ & $3(8)$ \\
\hline \multicolumn{3}{|l|}{ CellSearch CTC } \\
\hline CTC negative & $13(20)$ & $7(20)$ \\
\hline CTC positive & $11(11)$ & $0(11)$ \\
\hline \multicolumn{3}{|l|}{ iFISH-CTC } \\
\hline $\mathrm{CTC}>4$ & $11(12)$ & $1(12)$ \\
\hline $\mathrm{CTC} \leq 4$ & $19(30)$ & $11(30)$ \\
\hline
\end{tabular}

Mean OLFM4 expression, $150.40 \mathrm{ng} / \mathrm{ml}$. Data are presented as the level of expression (number of cases). CTC, circulating tumor cell; iFISH, immunostaining-fluorescence in situ hybridization; IHC, immunohistochemical staining; TNM, tumor-node-metastasis; OLFM4, olfactomedin 4.

washed five times with the wash solution. A total of $90 \mu \mathrm{l}$ substrate solution was added to each well. The solution turned yellow with the addition of the stop solution. The absorbance was measure immediately at $450 \mathrm{~nm}$ with a microplate reader.

$S Y B R$-Green reverse transcription-quantitative polymerase chain reaction $(R T-q P C R)$. Total RNA of the tumor tissue samples and matched breast cancer peritumoral tissues was extracted by TRIzol. The first strand cDNA fragments were reverse transcribed using Radom Primers. DyNAmo flash
SYBR-Green qPCR kit and two internal controls (GAPDH and RPLPO) were used for analysis of OLFM4 gene expression. The primer sequences for GAPDH are as follows: Forward, 5'-GCCACATCGCTCAGACACC-3' and reverse, 5'-GATGGCAACAATATCCACTTTACC-3'. The primers for RPLPO were as follows: Forward, 5'-CATCCAGCAGGT GTTCGAC-3' and reverse, 5'-GGTAGCCAATCTGCAGAC AGAC-3'. The primers for OLFM4 were as follows: Forward, 5'-GCAGCTTAGGCAGCGGAG-3' and reverse, 5'-GGC AGGGAAACAGAGCACT-3'. q PCR was performed using the ABI StepOnePlus ${ }^{\mathrm{TM}}$ Real-Time PCR system at $95^{\circ} \mathrm{C}$ for $15 \mathrm{~min}$, followed by 40 cycles at $95^{\circ} \mathrm{C}$ for $15 \mathrm{sec}$ and at $60^{\circ} \mathrm{C}$ for $30 \mathrm{sec}$. The $2^{-\Delta \Delta \mathrm{Cq}}$ relative quantitation method was used to calculate the relative gene expression level (22). PCR for each sample were repeated 3 times. GAPDH and RPLOP served as the internal references for each specimen, and therefore each specimen produced two sets of $2^{-\Delta \Delta C q}$ data. With GAPDH and RPLOP as the internal references, $2^{-\Delta \Delta \mathrm{C} q}<1$ was defined as low expression, $2^{-\Delta \Delta \mathrm{Cq}}>1$ was defined as high expression. When $2^{-\Delta \Delta \mathrm{Cq}}>1$ with GAPDH as an internal reference, and $2^{-\Delta \Delta \mathrm{Cq}}<1$ with RPLOP as an internal reference, cases were defined as medium expression.

Immunohistochemical analysis. The slides were incubated at $60^{\circ} \mathrm{C}$ for $1 \mathrm{~h}$, followed by deparaffinization in xylene at a temperature of $37^{\circ} \mathrm{C}$ for $10 \mathrm{~min}, 3$ times, and rehydration as follows: 2 min in 100\% ethanol, 2 min in 95\% ethanol, 2 min in $80 \%$ ethanol, 2 min in 70\% ethanol, 5 min in tap water and $5 \mathrm{~min}$ in distilled water. Citrate buffer $(0.01 \mathrm{M} \mathrm{Na}$ citrate-citric acid) was used as the retrieval solution. Rehydrated sections were immersed in the retrieval solution and processed in a microwave oven with the power set at $750 \mathrm{~W}$ for three cycles (5 min each). Following the completion of the third cycle, sections were allowed to cool at room temperature for $20 \mathrm{~min}$ and then rinsed in distilled water and PBS. The slides were then treated with endogenous peroxidase blocking agent for $10 \mathrm{~min}$ at room temperature to quench endogenous peroxidase activity, and non-immune goat serum for $10 \mathrm{~min}$ at room temperature to block non-specific binding. The sections were incubated with rabbit anti-OLFM4 (cat. no. NBP2-24535; Novus Biologicals, LLC, Littleton CO, USA) with a dilution of $1: 50$ overnight at $4^{\circ} \mathrm{C}$. After washing three times $(5 \mathrm{~min}$ for each wash) with PBS, biotin-labeled goat anti-mouse/rabbit IgG was added as the secondary antibody and incubated $10 \mathrm{~min}$ at room temperature. Three times ( $5 \mathrm{~min}$ for each wash) washing with PBS, and streptavidin-biotin-peroxidase reagent were added, followed by $10 \mathrm{~min}$ incubating at room temperature. Then DAB color liquid was added. Blocking agent, goat serum, secondary antibody and streptavidin-biotin-peroxidase reagent were purchased as part of the Maxim UltraSensitive kit (KIT-9720 A-D) from Maxim Biotechnologies (Fuzhou, Fujian, China) and were used according to manufacturer's protocol. The sections were counterstained with hematoxylin for 3-10 min, followed by washing, dehydration and mounting. Finally, five randomly selected fields were visualized under an upright fluorescence microscope (TS100; Nikon Corporation, Tokyo, Japan).

Statistical analysis. All statistical analyses were performed using by SPSS software (version 19.0; IBM Corp., Armonk, 
NY, USA). Kolmogorov-Smirnov was used to test whether the datasets were normally distributed $(23,24)$. When making comparisons in normally distributed datasets containing two groups, Student's t-test was used, and Mann-Whitney U-test was used to analyze datasets with a non-normal distribution. When making comparison in datasets containing multiple groups, one-way analysis of variance was used for normally distributed datasets, and Kruskal Wallis test was used for non-normally distributed data sets. Pearson's correlation coefficient was calculated to elucidate the correlation between two datasets. GraphPad Prism ${ }^{\circledR}$ (version 5.01; GraphPad Software, Inc., La Jolla, CA, USA) was used to construct the graphs $\mathrm{P}<0.05$ was considered statistically significant.

\section{Results}

Plasma OLFM4 expression levels in peripheral blood. Levels of plasma OLFM4 expression in peripheral blood were analyzed in 86 cases, including 26 cases of healthy individuals, 6 cases of benign breast tumor and 54 cases of breast cancer (Table I). The test results in all of the specimens ranged from 67.26 to $304.89 \mathrm{ng} / \mathrm{ml}$ [mean, $150.40 \mathrm{ng} / \mathrm{ml}$; standard deviation (SD) 56.50]. For patients with breast cancer, the expression levels of plasma OLFM4 varied from 67.26 to $173.01 \mathrm{ng} / \mathrm{ml}$ (mean, $119.31 \mathrm{ng} / \mathrm{ml}$; SD, 27.28), which was lower compared with the expression level in healthy individuals (mean, 222.16 ng/ml; SD, 37.74) (Fig. 1; $\mathrm{P}<0.0001$ ). No significant difference was obtained in plasma OLFM4 levels between 6 benign breast tumor cases and breast cancer patients $(\mathrm{P}=0.749)$. A normal distribution of OLFM4 expression levels in plasma was demonstrated using the Kolmogorov-Smirnov test in 60 patients with breast disease, including 25 cases above mean value and 35 cases below mean value. All of the 26 healthy individuals had higher plasma OLFM4 expression levels (160.49-304.89 ng/ml) compared with patients with breast cancer. With the exception of two healthy control specimens with relatively low values (160.49 and $162.70 \mathrm{ng} / \mathrm{ml}$ ), the mean value expression levels of plasma OLFM4 in all of the remaining control specimens were higher compared with the maximum value $(173.01 \mathrm{ng} / \mathrm{ml})$ in the patients with breast cancer. Plasma OLFM4 expression levels in peripheral blood indicated significant differences between healthy individuals and breast cancer patients, suggesting that the plasma OLFM4 level can be used as a supplementary indicator in breast cancer diagnosis.

Plasma OLFM4 level in breast cancer patients and its association with OLFM4 protein expression in tumor tissues. Tissue specimens of breast cancer resected following surgery were obtained from 28 patients among those enrolled for plasma OLFM4 detection. Following immunohistochemistry, one specimen, with low plasma OLFM4 level of $67.26 \mathrm{ng} / \mathrm{ml}$, was negative for OLFM4 protein expression. The remaining enrolled patients were divided into four groups according to OLFM4 protein expression levels in tumor tissues: $0,1+, 2+$, and $3+$ (Fig. 2). The OLFM4 protein expression level was $1+$ in 6 cases (plasma OLFM4 level, $88.52 \pm 17.41 \mathrm{ng} / \mathrm{ml}$ ), $2+$ in 13 cases (plasma OLFM4 level, 109.22 $\pm 12.34 \mathrm{ng} / \mathrm{ml}$ ), and $3+$ in 8 cases (plasma OLFM4 level, 129.92 $\pm 20.65 \mathrm{ng} / \mathrm{ml}$ ). Significant differences were observed among these four groups
A

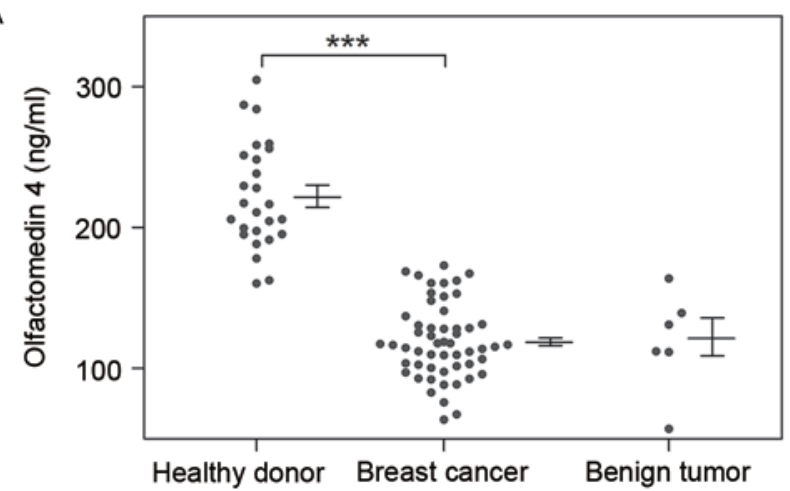

B

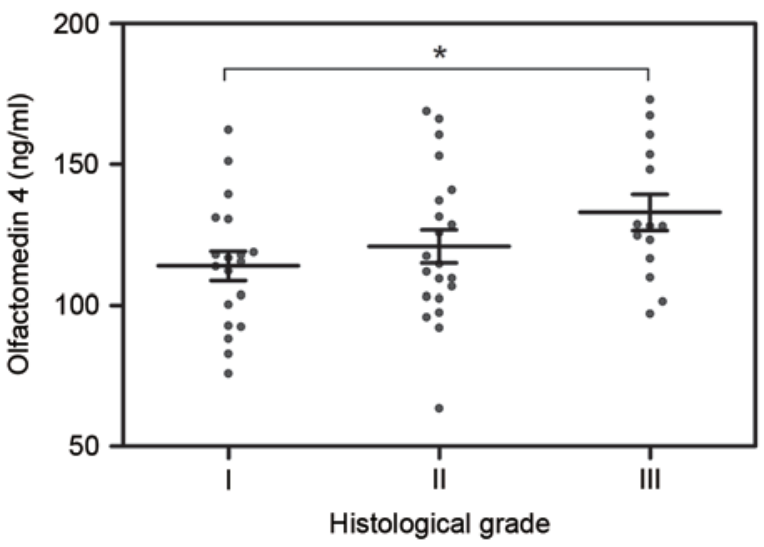

C

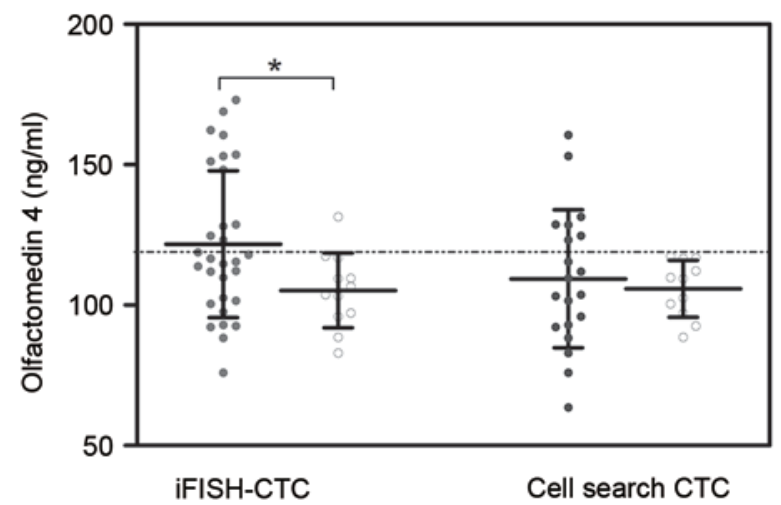

Figure 1. Detection of OLFM4 protein in plasma samples by ELISA (A) Plasma OLFM4 level in breast cancer patients, benign tumor and healthy donor. The level of plasma OLFM4 in patients with breast cancer was significantly different compared with the level in healthy donors. (B) Plasma OLFM4 level in breast cancer with different histological grades. (C) Association of plasma OLFM4 with CTC enumeration according to two methods of CTC detection. ${ }^{*} \mathrm{P}<0.05,{ }^{* * *} \mathrm{P}<0.001$. CTC, circulating tumor cell; iFISH, immunostaining-fluorescence in situ hybridization; OLFM4, olfactomedin 4.

$(\mathrm{P}<0.0001)$. For each group, the mean plasma OLFM4 level was in a significant linear correlation with the OLFM4 protein level in tumor tissues $\left(\mathrm{R}^{2}=1\right.$; Fig. 2) This result demonstrated the consistency between the plasma OLFM4 level in peripheral blood and OLFM4 protein expression in the tumor tissue of breast cancer patients.

Plasma OLFM4 expression level in peripheral blood and clinicopathological features in patients with breast cancer. The 54 breast cancer patients included 19 cases of 


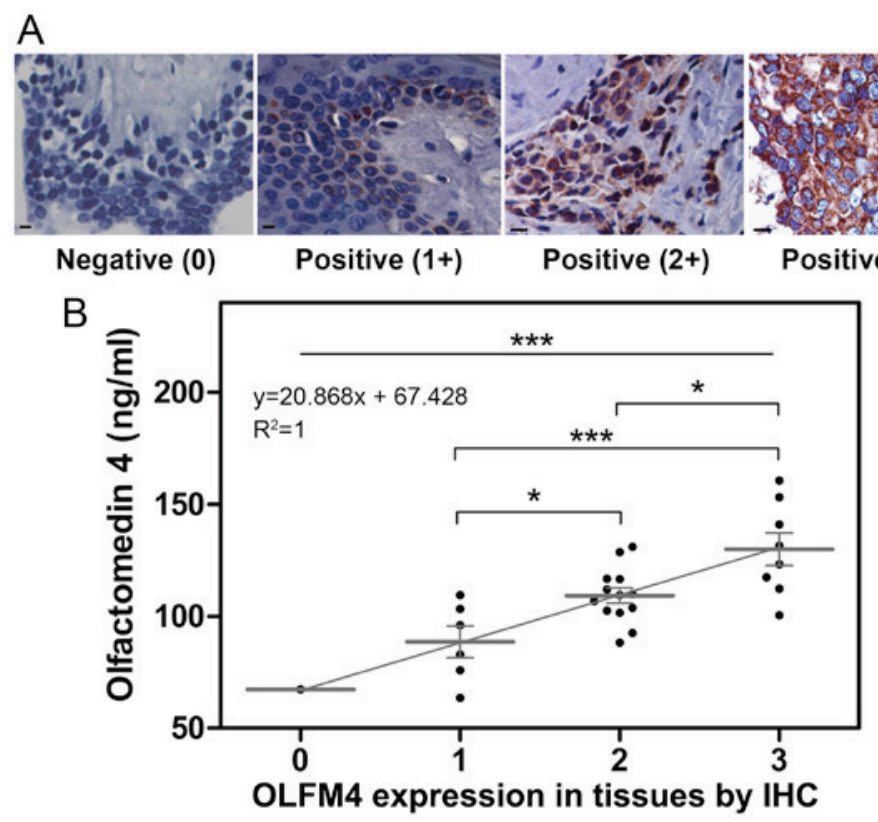

Figure 2. Levels of plasma OLFM4 expression in peripheral blood and OLFM4 protein in tumor tissues. (A) Different OLFM4 expression levels in the cancer tissues. Negative (0) indicates no expression of OLFM4; positive (1+) indicates low expression of OLFM4; positive (2+) indicates moderate expression of OLFM4; and positive (3+) indicates high expression of OLFM4. Original magnification, $\mathrm{x} 400$. (B) Comparative analysis of OLFM4 protein levels in plasma and tissues. "P<0.05. IHC, immunohistochemistry; OLFM4, olfactomedin 4.

histological grade I, with low plasma OLFM4 expression levels $(113.91 \pm 22.70 \mathrm{ng} / \mathrm{ml}) ; 21$ cases of histological grade II, with slightly increased plasma OLFM4 expression levels $(120.83 \pm 26.80 \mathrm{ng} / \mathrm{ml})$; and 14 cases of histological grade III, with the high plasma OLFM4 expression levels $(132.90 \pm 24.03 \mathrm{ng} / \mathrm{ml})$. There was no significant difference in the plasma OLFM4 expression level among breast cancer patients with different histological grades $(\mathrm{P}=0.138)$. Additionally, there was no difference between patients of grades II and I $(\mathrm{P}=0.507)$ or grades II and III $(\mathrm{P}=0.201)$ either. However, a significant difference in the plasma OLFM4 expression level was observed between patients of grades III and $\mathrm{I}(\mathrm{P}=0.041$; Fig. 1B).

The associations between plasma OLFM4 levels and other clinicopathological features, including lymph node status, progesterone/estrogen receptor and TNM stage in patients with breast cancer were also analyzed. Generally, no significant associations were observed between plasma OLFM4 levels and these clinicopathological features (Fig. 3).

Plasma OLFM4 expression level and CTC number in the peripheral blood of patients with breast cancer. In total, 42 samples from patients with breast cancer and 5 benign samples underwent CTC detection by iFISH-subtraction enrichment. Among 12 patients with $>4$ CTCs per $7.5 \mathrm{ml}$ peripheral blood, the plasma OLFM4 level was above mean value in only 1 case $(130.58 \mathrm{ng} / \mathrm{ml})$. Plasma OLFM4 levels were below mean value in the remaining 11 cases $(88.53-117.45 \mathrm{ng} / \mathrm{ml})$. CTC detection revealed a significant difference between breast cancer patients with plasma OLFM4 levels below and above mean value (median 3 CTCs per $7.5 \mathrm{ml}$ plasma blood vs. 2.5 CTCs per $7.5 \mathrm{ml}$ plasma blood), and 11 cases were below average $(\mathrm{P}<0.05$; Fig. 1; Table $\mathrm{I})$. Moreover, 31 patients with breast cancer underwent CTC detection using the CellSearch system. Among the 11 CTC-positive patients ( 1 or 2 CTCs per $7.5 \mathrm{ml}$ peripheral blood), plasma OLFM4 expression remained below mean value. For the other 20 CTC-negative patients, plasma OLFM4 expression was above mean value in 7 cases and below mean value in 13 cases. These results implied that CTC-positive breast cancer patients were associated with low plasma OLFM4 expression (Fig. 1; Table I). None of the 5 benign samples were detected to be CTC-positive in the present study. A total of 2 samples with $1 \mathrm{CTC}$ and 3 samples with 0 CTC were detected in $7.5 \mathrm{ml}$ peripheral blood by iFISH-subtraction enrichment, and all 5 samples with 0 CTC were detected by CellSearch system.

OLFM4 gene expression level in breast cancer tumor tissues. A quantitative analysis of OLFM4 expression at the mRNA level was undertaken in 24 pairs of tumor tissue and normal adjacent tissue specimens. The expression level of OLFM4 relative to the internal reference in tissue specimens was calculated based on the $\Delta \mathrm{Cq}$ value of OLFM4 gene amplification relative to the $\Delta \mathrm{Cq}$ value of internal reference gene amplification, i.e., $2^{-\Delta \mathrm{Cq}}$. OLFM4 expression in tumor tissues relative to normal adjacent tissues was calculated using the $2^{-\Delta \Delta \mathrm{Cq}}$ method. GAPDH and RPLOP served as the internal references for each specimen, and therefore each specimen produced two sets of $2^{-\Delta \Delta \mathrm{Cq}}$ data. With GAPDH and RPLOP as the internal references, $2^{-\Delta \Delta \mathrm{Cq}}<1$ was detected in 7 cases for OLFM4 in tissue specimens, which indicated that OLFM4 expression was lower in 7 tumor tissue specimens compared with normal adjacent tissues. $2^{-\Delta \Delta \mathrm{Cq}}>1$ was detected in 8 cases for OLFM4 in tissue specimens, which indicated that OLFM4 expression was higher in 8 tumor tissue samples compared with normal adjacent tissues. For the remaining 9 cases, OLFM4 expression was higher in tumor tissues with GAPDH as an internal reference, while the value was higher in normal 

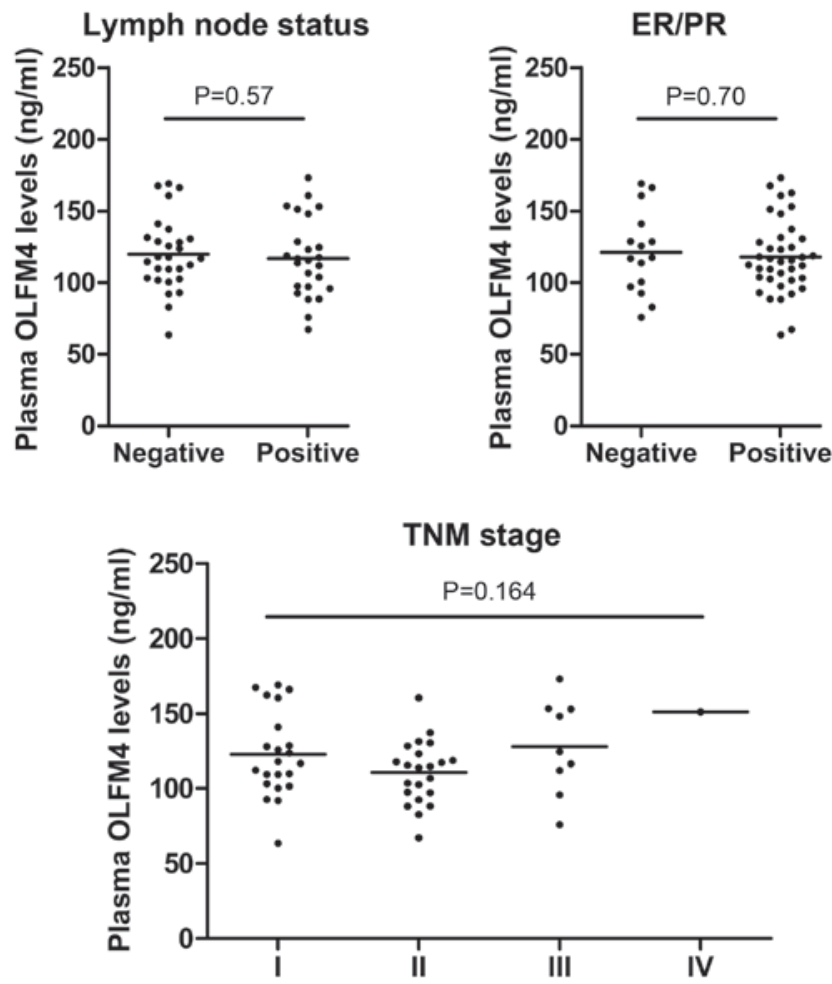

Figure 3. Levels of plasma OLFM4 expression in peripheral blood and clinicopathological features of patients with breast cancer. Horizontal lines represent the median values. ER/PR, progesterone/estrogen receptor; OLFM4, olfactomedin 4; TNM, tumor-node-metastasis.

adjacent tissues with RPLOP as an internal reference. Based on the results of $2^{-\Delta \Delta \mathrm{Cq}}$ with the two internal references, the 24 patients were further divided into three groups: High OLFM4 expression (8 cases), medium OLFM4 expression (9 cases) and low OLFM4 expression (7 cases).

In terms of histological grade, the 24 breast cancer patients included 3 cases of grade I, 13 cases of grade II and 8 cases of grade III. With GAPDH as the internal control, the mean value OLFM4 expression levels were highest in patients with grade I tumors and lowest in patients with grade III tumors (Fig. 4). However, the test results were not significantly different between the three groups of different tumor grades $(\mathrm{P}=0.411)$. With RPLOP as the internal control, the mean value OLFM4 expression level was significantly higher in patients with grade III tumors compared with those with grade I tumors ( $\mathrm{P}=0.026$; Fig. 4). There was no significant difference in OLFM4 expression between patients with grade II tumors and grades II and III.

\section{Discussion}

OLFM4 is primarily expressed in the bone marrow, stomach, small intestine, colon, pancreas and prostate tissues $(2,3)$. This molecular marker may have significance in tumor diagnosis, treatment and prognosis. It has been shown that the OLFM4 expression level in tissue specimens has a close association with tumor progression. OLFM4 expression is abnormal at the mRNA and protein levels in the majority of tumor tissues in gastric $(11,25)$, colorectal $(5,10)$, lung $(10)$, cervical (12) and prostate cancer (13). Such changes may result in abnormalities

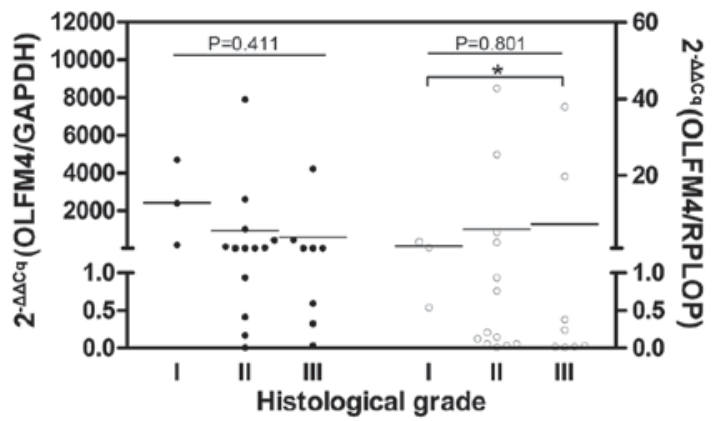

Figure 4. mRNA expression of OLFM4 in tumor tissues. mRNA expression of OLFM4 gene was quantified with two reference genes of GAPDH and RPLOP separately in each sample. $2^{-\Delta \Delta \mathrm{Cq}}$ indicates OLFM4 gene expression in tumor tissues relative to the adjacent normal tissues. Y-axis on the left side represents the relative expression of OLFM4 calculated with GAPDH. Y-axis on the right side represents the relative expression of OLFM4 calculated with RPLOP. ${ }^{*} \mathrm{P}<0.05$. OLFM4, olfactomedin 4.

in OLFM4 regulation of the biological behavior of cells (e.g., cell adhesion, cycle and apoptosis), leading to tumor development and progression (4). However, the vast majority of relevant studies $(10,11,13,25)$ are based on gene or protein expression analysis using tumor tissue specimens. As a consequence, such research is largely limited in cases where collection of clinical specimens was difficult from surgery.

OLFM4 is a secreted glycoprotein (26). A study analyzed serum OLFM4 levels in the peripheral blood of patients with gastric cancer prior to surgery (11), and the results showed that OLFM4 expression in peripheral blood may be used as an indicator for early diagnosis and prognostic determination of gastric cancer. To date, serum/plasma OLFM4 expression patterns in other tumors, including breast cancer, have not yet been reported. In the present study, the expression levels of plasma OLFM4 in peripheral blood was consistent with the levels of OLFM4 protein expression in the corresponding tumor tissues of 28 patients with breast cancer. There was a linear correlation between plasma OLFM4 expression levels in peripheral blood and OLFM4 expression level groups (high/medium/low) in tissue specimens $(\mathrm{R}=1.000)$. These results suggest that the plasma OLFM4 expression characteristics in the peripheral blood of breast cancer patients represent the OLFM4 expression patterns in tumor tissue specimens of breast cancer. Such data can be applied for OLFM4 expression analysis in cases where collection of tumor tissue specimens is difficult.

In the present study, the plasma OLFM4 expression level differed markedly between breast cancer patients and healthy controls, indicating that it can be used as a supplementary indicator in tumor diagnosis. A study has reported that OLFM4 levels in peripheral blood are significantly higher in gastric cancer patients prior to surgery compared with normal individuals (9). An opposite trend was observed in the present study, that is, the plasma OLFM4 expression levels were far lower in breast cancer patients compared with healthy controls. Additionally, the mean value plasma OLFM4 expression level in healthy controls $(222.16 \pm 37.74 \mathrm{ng} / \mathrm{ml})$ was markedly higher compared with the values reported in the gastric cancer study $(16.6 \pm 1.6 \mathrm{ng} / \mathrm{ml})$, which perhaps is associated with the use of different testing reagents. Research 
of OLFM4 expression in tissue specimens has shown that OLFM4 is expressed at relatively low levels in normal tissues, while the expression is upregulated in tumor tissues in the colon and pancreas. Furthermore, there is a loss of OLFM4 expression in prostate cancer tumor tissues but high expression in normal tissues (13). These findings demonstrate that OLFM4 expression follows different patterns in various tumors. It has been reported that OLFM4 mRNA expression is upregulated in the majority of tumor tissue specimens in colon cancer (90\%) and lung cancer (85\%) relative to normal adjacent tissue, whereas $69 \%$ of tumor specimens in breast cancer are associated with upregulated OLFM4 expression (10). In the present study, OLFM4 mRNA expression was analyzed in matched specimens of tumor and normal adjacent tissues. Of the 24 patients, only 8 cases (RPLOP as internal control) or 17 cases (GAPDH as internal control) exhibited higher OLFM4 expression in tumor tissues compared with normal adjacent tissues, in agreement with the previous report. The OLFM4 expression patterns in breast cancer are opposite to those observed in the colon (5) and pancreas (27), but similar to the patterns in prostate cancer (13). This phenomenon may be due to the cell and tissue specificity of the role of OLFM4. However, various reference genes have been used in different studies, and the expression of reference genes in tumor tissues may change to some extent $(28,29)$, resulting in biased test results. Therefore, it is recommended that gene expression analysis should be performed in tumor tissue specimens using multiple internal reference genes for comparison. In the present study, there was no difference in plasma OLFM4 levels between patients with benign ( 6 cases) and malignant tumors. Therefore, OLFM4 expression cannot be used to distinguish between benign and malignant breast tumors.

OLFM4 has a complex role in tumors, and there are large differences in expression with different degrees of malignancy in the same tumor. It has been previously reported that pancreatic cancer with higher OLFM4 gene expression has increased malignant behavior and invasive capacity as well as the ability to metastasize more easily (30). OLFM4 expression is upregulated in early colorectal and gastric cancer but downregulated in progressive or advanced tumors $(2,31)$. OLFM4 expression is negatively correlated with invasion depth, lymph node metastasis and tumor clinical stage (2). OLFM4 expression in undifferentiated or poorly differentiated tumor tissue is significantly lower compared with expression in well-differentiated tumor tissue of gastric cancer. Moreover, OLFM4 expression is lower in diffuse-type compared with intestinal-type gastric cancer. Therefore, OLFM4 expression can be used as an indicator in the prognostic determination of this type of cancer (32). The results from the present study indicated that plasma OLFM4 levels in breast cancer patients were associated with histological tumor differentiation. High plasma OLFM4 levels were detected in patients with high histological grade, which was consistent with data from previous studies on pancreatic (5) and gastric cancer $(9,11)$.

GADPH has been reported to be expressed at a relatively high level in the gene expression study of some types of cancer $(33,34)$, including lung, kidney and breast cancer. Otherwise, the expression of RPLPO may be more constant in some tissues, including lung, ovarian and breast cancer $(34,35)$. Published gene expression studies have predominantly found that the parallel determination of two or more housekeeping genes in a given sample population or experimental condition is crucial to gene expression analysis in cancer studies (36). In the present study, different conclusions were drawn with different reference genes in the analysis of OLFM4 mRNA expression in tumor tissue from breast cancer patients. This finding also indicates that additional caution is required when selecting reference genes for gene expression analysis in tumor tissue specimens. The test results with the RPLOP gene as an internal reference were statistically significant and in agreement with the trends in plasma OLFM4. Therefore, high OLFM4 expression in plasma or tissue specimens of breast cancer patients is more likely to represent poor histological differentiation and increased invasive/metastatic capabilities. However, low OLFM4 expression indicates low histological grade and decreased invasive/metastatic capabilities.

In the present study, CTC detection was undertaken in a portion of the enrolled patients. CTC detection by iFISH-subtraction enrichment has a higher detection rate compared with the CellSearch system by EpCAM-positive enrichment (19). Nevertheless, the two methods and plasma OLFM4 levels are common in that CTC-positive patients have lower levels of OLFM4 in plasma compared with CTC-negative patients. To date, to the best our knowledge, no study has assessed the correlation between the number of CTCs and OLFM4 expression. It has been hypothesized that CTCs are associated with metastatic potential and prognosis of tumors. This hypothesis suggests that OLFM4 expression is associated with tumor metastasis and prognosis in patients with breast cancer, which is inconsistent with the association observed between histological grade and OLFM4 level. One possibility is that all of the patients enrolled in the present study had no tumor metastases, and the overall percentage of CTC-positive patients and detected values of CTCs were low, resulting in biased statistical results. However, previous studies suggest that OLFM4 may facilitate tumorigenesis in early tumors by anti-apoptotic effects. By contrast, OLFM4 overexpression is also able to promote cell adhesion by interacting with lectin or cadherin (37), thereby inhibiting tumor progression and metastasis. Therefore, a low level of OLFM4 expression is able to inhibit adhesion between cells (8) and thereby promote CTC generation. Therefore, to investigate the detailed associations between OLFM4 level and tumor metastasis in patients with breast cancer, a larger number of tumor specimens representing different progression stages need to be analyzed longitudinally to examine the changes and roles of OLFM4 expression in tumor development and progression.

\section{Acknowledgments}

The present study was supported by the National Natural Science Foundation of Shanghai (grant no. 12ZR1438700), the National Natural Science Foundation of China (grant nos. 81300684 and 81502546) and Pudong Science and Technology Commission, and Science and Technology Innovation Action Project. 


\section{References}

1. Grover PK, Hardingham JE and Cummins AG: Stem cell marker olfactomedin 4: Critical appraisal of its characteristics and role in tumorigenesis. Cancer Metastasis Rev 29: 761-775, 2010.

2. Kulkarni NH, Karavanich CA, Atchley WR and Anholt RR: Characterization and differential expression of a human gene family of olfactomedin-related proteins. Genet Res 76: 41-50, 2000.

3. Zhang J, Liu WL, Tang DC, Chen L, Wang M, Pack SD, Zhuang Z and Rodgers GP: Identification and characterization of a novel member of olfactomedin-related protein family, hGC-1, expressed during myeloid lineage development. Gene 283: 83-93, 2002.

4. Tomarev SI and Nakaya N: Olfactomedin domain-containing proteins: Possible mechanisms of action and functions in normal development and pathology. Mol Neurobiol 40: 122-138, 2009.

5. Besson D, Pavageau AH, Valo I, Bourreau A, Bélanger A, Eymerit-Morin C, Moulière A, Chassevent A, Boisdron-Celle M, Morel A, et al: A quantitative proteomic approach of the different stages of colorectal cancer establishes OLFM4 as a new nonmetastatic tumor marker. Mol Cell Proteomics 10 M111.009712, 2011.

6. Huang Y, Yang M, Yang H and Zeng Z: Upregulation of the GRIM-19 gene suppresses invasion and metastasis of human gastric cancer SGC-7901 cell line. Exp Cell Res 316: 2061-2070, 2010.

7. Kobayashi D, Koshida S, Moriai R, Tsuji N and Watanabe N: Olfactomedin 4 promotes S-phase transition in proliferation of pancreatic cancer cells. Cancer Sci 98: 334-340, 2007.

8. Liu W, Liu Y, Zhu J, Wright E, Ding I and Rodgers GP: Reduced hGC-1 protein expression is associated with malignant progression of colon carcinoma. Clin Cancer Res 14: 1041-1049, 2008

9. Park KS, Kim KK, Piao ZH, Kim MK, Lee HJ, Kim YC, Lee KS, Lee JH and Kim KE: Olfactomedin 4 suppresses tumor growth and metastasis of mouse melanoma cells through downregulation of integrin and MMP genes. Mol Cells 34 555-561, 2012.

10. Koshida S, Kobayashi D, Moriai R, Tsuji N and Watanabe N: Specific overexpression of OLFM4 (GW112/HGC-1) mRNA in colon, breast and lung cancer tissues detected using quantitative analysis. Cancer Sci 98: 315-320, 2007.

11. Oue N, Sentani K, Noguchi T, Ohara S, Sakamoto N, Hayashi T, Anami K, Motoshita J, Ito M, Tanaka S, et al: Serum olfactomedin 4 (GW112, hGC-1) in combination with Reg IV is a highly sensitive biomarker for gastric cancer patients. Int J Cancer 125: 2383-2392, 2009.

12. Yu L, He M, Yang Z, Chen G, Li M, Wang L and Chen S: Olfactomedin 4 is a marker for progression of cervical neoplasia. Int J Gynecol Cancer 21: 367-372, 2011.

13. Chen L, Li H, Liu W, Zhu J, Zhao X, Wright E, Cao L, Ding I and Rodgers GP: Olfactomedin 4 suppresses prostate cancer cell growth and metastasis via negative interaction with cathepsin D and SDF-1. Carcinogenesis 32: 986-994, 2011.

14. Yan H, Lu D, Xu L, Xie Q, Dong X and Wu Y: Increased expression level of Olfactomedin4 in peripheral blood mononuclear cells of pancreatic adenocarcinoma patients. Hepatogastroenterology 58: 1354-1359, 2011.

15. Huang MY, Wang HM, Chang HJ, Hsiao CP, Wang JY and Lin SR: Overexpression of S100B, TM4SF4, and OLFM4 genes is correlated with liver metastasis in Taiwanese colorectal cancer patients. DNA Cell Biol 31: 43-49, 2012.

16. Huang MY, Wang HM, Tok TS, Chang HJ, Chang MS, Cheng TL, Wang JY and Lin SR: EVI2B, ATP2A2, S100B, TM4SF3, and OLFM4 as potential prognostic markers for postoperative Taiwanese colorectal cancer patients. DNA Cell Biol 31: 625-635, 2012.

17. Xiong B, Lei X, Zhang L and Fu J: The clinical significance and biological function of olfactomedin 4 in triple negative breast cancer. Biomed Pharmacother 86: 67-73, 2017.
18. Amin MB, Edge SB, Greene FL, Schilsky RL, Gaspar LE, Washington MK, Sullivan DC, Brookland RK, Brierley JD, Balch CM, et al (eds): AJCC (American Joint Committee on Cancer) Cancer Staging Manual, 8th edition, Springer, Chicago, 2017.

19. Li Y, Zhang X, Ge S, Gao J, Gong J, Lu M, Zhang Q, Cao Y, Wang DD, Lin PP and Shen L: Clinical significance of phenotyping and karyotyping of circulating tumor cells in patients with advanced gastric cancer. Oncotarget 5: 6594-6602, 2014.

20. Zhang Y, Wang F, Ning N, Chen Q, Yang Z, Guo Y, Xu D, Zhang D, Zhan T and Cui W: Patterns of circulating tumor cells identified by CEP8, CK and CD45 in pancreatic cancer. Int J Cancer 136: 1228-1233, 2015.

21. Cristofanilli M, Budd GT, Ellis MJ, Stopeck A, Matera J, Miller MC, Reuben JM, Doyle GV, Allard WJ, Terstappen LW and Hayes DF: Circulating tumor cells, disease progression, and survival in metastatic breast cancer. N Engl J Med 351: 781-791, 2004.

22. Livak KJ and Schmittgen TD: Analysis of relative gene expression data using real-time quantitative PCR and the 2(-Delta Delta C(T)) Method. Methods 25: 402-408, 2001.

23. Abayomi K, Gelman A and Levy M: Diagnostics for multivariate imputations. Series C Appl Stat 57: 273-291, 2008.

24. Conover WJ: Practical nonparametric statistics. 2nd edition. New York, Wiley, 1980.

25. Yu L, Wang L and Chen S: Olfactomedin 4, a novel marker for the differentiation and progression of gastrointestinal cancers. Neoplasma 58: 9-13, 2011.

26. Snyder DA, Rivers AM, Yokoe H, Menco BP and Anholt RR: Olfactomedin: Purification, characterization, and localization of a novel olfactory glycoprotein. Biochemistry 30: 9143-9153, 1991.

27. Liu W, Yan M, Liu Y, Wang R, Li C, Deng C, Singh A, Coleman WG Jr and Rodgers GP: Olfactomedin 4 down-regulates innate immunity against Helicobacter pylori infection. Proc Natl Acad Sci USA 107: 11056-11061,2010.

28. Révillion F, Pawlowski V, Hornez L and Peyrat JP: Glyceraldehyde-3-phosphate dehydrogenase gene expression in human breast cancer. Eur J Cancer 36: 1038-1042, 2000.

29. Radonić A, Thulke S, Mackay IM, Landt O, Siegert W and Nitsche A: Guideline to reference gene selection for quantitative real-time PCR. Biochem Biophys Res Commun 313: 856-862, 2004.

30. Takadate T, Onogawa T, Fukuda T, Motoi F, Suzuki T, Fujii K, Kihara M, Mikami S, Bando Y, Maeda S, et al: Novel prognostic protein markers of resectable pancreatic cancer identified by coupled shotgun and targeted proteomics using formalin-fixed paraffin-embedded tissues. Int J Cancer 132: 1368-1382, 2013.

31. Liu RH, Yang MH, Xiang H, Bao LM, Yang HA, Yue LW, Jiang X, Ang N, Wu LY and Huang Y: Depletion of OLFM4 gene inhibits cell growth and increases sensitization to hydrogen peroxide and tumor necrosis factor-alpha induced-apoptosis in gastric cancer cells. J Biomed Sci 19: 38, 2012.

32. Suh YS, Lee HJ, Jung EJ, Kim MA, Nam KT, Goldenring JR, Yang HK and Kim WH: The combined expression of metaplasia biomarkers predicts the prognosis of gastric cancer. Ann Surg Oncol 19: 1240-1249, 2012.

33. de Kok JB, Roelofs RW, Giesendorf BA, Pennings JL, Waas ET, Feuth T, Swinkels DW and Span PN: Normalization of gene expression measurements in tumor tissues: Comparison of 13 endogenous control genes. Lab Invest 85: 154-159, 2005.

34. Lyng MB, Laenkholm AV, Pallisgaard $\mathrm{N}$ and Ditzel HJ: Identification of genes for normalization of real-time RT-PCR data in breast carcinomas. BMC Cancer 8: 20, 2008.

35. Gresner P, Gromadzinska J and Wasowicz W: Reference genes for gene expression studies on non-small cell lung cancer. Acta Biochim Pol 56: 307-316, 2009.

36. Caradec J, Sirab N, Keumeugni C, Moutereau S, Chimingqi M, Matar C, Revaud D, Bah M, Manivet P, Conti M and Loric S: 'Desperate house genes': The dramatic example of hypoxia. Br J Cancer 102: 1037-1043, 2010.

37. Liu W, Chen L, Zhu J and Rodgers GP: The glycoprotein hGC-1 binds to cadherin and lectins. Exp Cell Res 312: 1785-1797, 2006. 